Vol. 9, no. 18, 2018, pp. 127-139

ISSN 2007-1868

\title{
BREVE MEDITACIÓN SOBRE LA VIOLENCIA
}

José Alberto Mora Zamorano

Facultad de Ciencias Humanas y Sociales

Universidad La Salle

RESUMEN: El presente artículo presenta una breve meditación sobre la violencia a partir de las nociones psicoanalíticas de trauma y acontecimiento límite para cuestionar la base de los dispositivos narrativos en que se construye su memoria tanto como historia y testimonio. El problema se plantea a partir de la patologización de las representaciones en el testimonio, y la distancia crítica con respecto a las representaciones resistentes. En esa toma de distancia la representación de la agresión como instinto biológico se separa de la violencia constituida en el ámbito simbólico de la ley. Sobre esta distinción se genera un cuestionamiento al posicionamento subjetivo de víctima.

PAlABRAS ClAVE: Trauma - acontecimiento límite - testimonio - memoria agresión · violencia

ABSTRACT: This article presents a brief meditation on violence from the psychoanalytic notions of trauma and limit-experience to question the basis of the narrative devices in which their memory is built as well as history and testimony. The problem arises from the pathologization of the representations in the testimony, and the critical distance with the resistant representations. In this distance, the representation of aggression as a biological instinct is separated from the violence constituted in the symbolic sphere of the law. This distinction oblige to question the subjective positioning of the victim.

KEYWORDS: Trauma - Limit-Experience - Testimony $\cdot$ Memory $\cdot$ Aggression · violence 


\section{Introducción}

¿Cómo preguntarse sobre la violencia? ¿Cómo hacerlo desde la Filosofía? Esas preguntas más que exigir respuestas definitivas invitan a la reflexión, y toda reflexión parte del regreso del ser, del acto o acontecimiento al sujeto. El sujeto colectivo o individuado; colectivo porque la reflexión sobre la violencia funda historia, memoria colectiva de la cual participa un balbuceante "Yo" que se sabe individuado. No es un individuo como tal sino que se sabe distinto de lo que lo configura: su modo de participar de la memoria. Cada reflexión es una individuación de las subjetividades o sujeciones colectivas. Cada meditación es la reflexión que busca desaprenderse de todas las sujeciones, incluida la del sí mismo, y abandonarse a la cosa misma.

Pero la violencia exige más bien lo contrario, no el abandono sino el regreso a sí, al Yo. La violencia acontece y por lo tanto se padece o se ejerce y por lo tanto se causa; en el primer caso, apelamos al dolor desde la memoria, en el segundo, a la ética. En esta breve reflexión sobre la violencia trataremos de dibujar un hilo conductor que va desde el pathos al ethos de la violencia, y, en tanto su reflexión exige la parcialidad de las sujeciones, se valdrá de las reflexiones que otros han realizado sobre el tema para intentar plantear, acaso, preguntas.

En la primera parte ahondaremos en los dispositivos configuradores de la memoria y su relación con la historia como reconstrucción de la violencia como acontecimiento-límite; esto es, la violencia como padecimiento. En la segunda parte revisaremos la distinción planteada entre la agresión y la violencia, partiendo del impulso natural de cada organismo como un factor no determinante de la experiencia de la segunda, y relacionarla con el aspecto de lo simbólico como su distinción antropógena. En un tercer apartado revisaremos esta consideración de la violencia como aquélla que funda derecho, en un sentido amplio, incluso, aquella que culturalmente establece leyes y distinciones. De este modo trazamos un hilo conductor entre la violencia como padecimiento a la violencia como principio de codificación moral o ética. Por último, se dejan unas reflexiones finales sobre este tipo de violencia, su pertinencia con relación a los dispositivos configuradores de su patologización así como su cuestionamiento y crítica, para concluir con un abierto cuestionamiento. 


\section{Trauma: historia y testimonio del acontecimiento límite}

Cuando hablamos o reflexionamos sobre la violencia lo hacemos con posterioridad y previsión; tratamos de reconstruir o comprender un evento traumático y evitar su repetición. Para el historiador Dominick LaCapra una de sus principales preocupaciones ha sido la relación entre historia y psicoanálisis como forma de evitar la patologización de las representaciones que reconstruyen esa memoria colectiva (2009 p. 207). Para este historiador la posibilidad de trazar un vínculo entre estas dos disciplinas se orienta por preocupaciones éticas y políticas, y para reconstruir una teoría crítica propia del ejercicio del historiador en la reconstrucción de la memoria. ${ }^{1}$

La imaginación juega un papel central en este ejercicio de reconstrucción y, a veces, ofrece un alivio momentáneo o vía de escape pero permanecen restos traumáticos que resisten el poder curador de este ejercicio (p. 208). Para LaCapra la reconstrucción histórica tiene un fin terapéutico, libra de la situación límite, permite articularla por medio del lenguaje, en la reconstrucción de una memoria que utiliza la imaginación para representar el recuerdo; de ahí los riesgos de patologización y la resistencia de algunos recuerdos (p. 208 y ss). La memoria, o el ejercicio de su reconstrucción como una terapia ético-política, tiene que poner estos recuerdos que se resisten en perspectiva, ponerlos a prueba a partir de un ejercicio de codificación, de simbolización en la estructuración de la memoria; esto es, tratarle de darles un cauce o replantearlos a la luz de nuevas narrativas (2009).

En este contexto, los recuerdos puestos críticamente a prueba pueden aparecer como el necesario punto de partida de toda actividad simbólica, aún cuando estén constantemente amenazados por lapsus, huecos o distorsiones. La cuestión de la memoria debe ocupar un lugar prominente en cualquier acontecimiento, un lugar que puede incluso ser exagerado precisamente a causa de la dificultad de recordar hechos que desafían a la imaginación y que los métodos convencionales de representación no pueden abarcar por completo (2009, p.209).

Según este autor, la indagación crítica de los recuerdos tendría como fin controlar los recuerdos y permitir el acceso a los acontecimientos

${ }^{1}$ Cf. Ibid., y LaCapra 2016. 
límites, los cuales "implican brechas, distorsiones y limitaciones al menos respecto de la experiencia del trauma en sí" (pp. 209-210).

El ejercicio del historiador que parte de una teoría crítica, y que se orienta en función de problemas éticos y políticos, busca en la reconstrucción de la memoria sobreponerse a los recuerdos de difícil acceso. Busca llenar los intersticios y eliminar las distorsiones que generan la permanencia del trauma para sobreponerse a éste; la posibilidad de recontar, de volver a narrar el acontecimiento que causó el trama tiene un fin liberador: terapéutico, ético y político. Y representa no sólo la posibilidad de ejercer un control sobre los recuerdos que tiende a patoligizarse, a fantasmátizarse al punto de poseer la memoria e impedir por completo la superación de acontecimiento límite (pp. 212-213). "En este sentido, la memoria puede convertirse en una forma de recuperar rumbos perdidos y alejarse críticamente de los aspectos menos deseables del pasado así como para intentar honrar otros o convertirlos en bases de una acción en el presente y el futuro" (2009).

En tanto se trata de superar el trauma surge entonces la necesidad de incorporar en la reconstrucción de la memoria nociones centrales del psicoanálisis como el trabajo del duelo o el pasaje al acto (2009). Sin embargo, en tanto se trata del trabajo de reconstrucción por parte del historiador, y de un trabajo crítico que no quede presa de las espectralidades de los recuerdos que se resisten al trabajo terapéutico, LaCapra se pregunta (en un trabajo posterior) por los límites reconocibles en el trabajo de reconfiguración de la memoria, en aquellos elementos del goce lacaniano, y que Freud ha codificado como las pulsiones propias de la vida (LaCapra 2016, p. 5):

¿Cómo puede una historiografía crítica reconocer y dar cuenta de manera sensible, de las fuerzas compulsivas, típicamente violentas, destructivas y en el mejor de los casos extremadamente ambivalentes, que tan notable papel ha desempeñado en los llamados acontecimientos y experiencias límite de la historia? ¿Acaso el intento de hacer tal cosa convierte necesariamente al investigador en inadvertido cómplice de los objetos de estudio, optando por la violencia, confundiendo compasión con identificación en incluso representado de manera trasferencial la repetición compulsiva? ¿Acaso un enfoque propio de los problemas requiere realizar o validar alguna case de exceso (negatividad absoluta, melancolía idealizada, una estética incondicional de lo sublime, una aprehensión apocalíptica cuando un deseo, la afirmación de la creación ex nihilo o la rotunda esperanza utópica)? ¿Cómo 
entender las recientes, y a veces misteriosas invocaciones a los postsecular a la relación humano-animal? ¿Qué reuqiere, en general, un intento de "pensar" la historia en relación con el complejo problema de los límites? (2009)

Los cuestionamientos de LaCapra son sugerentes en el plano de una epistemología de la historia o teoría crítica que pretende establecer un plano ético y político en la reconstrucción de la historia. Esta reconstrucción, como ya se mencionó líneas arrba, es terapéutica en tanto somete a crítica los recuerdos que se tienen sobre algún acontecimiento límite, su simbolización que se encuentra amenazada por una fantasmatización que mantiene en presente vivido, por lapsos, el hecho del trauma. Sin embargo, los cuestionamientos son más sugerentes para el tema que nos compete si esta reconstrucción de la memoria, que funciona en el plano de los tropos, de las formas lingüísitcas de las que se compone el testimonio que simboliza, en nuestro caso, la violencia como acontecimiento límite.

Esto es, si la patoligización de los recuerdos, las brechas, los lapsos se actualizan durante el trabajo acrítico del testimonio; y por lo tanto, la simbolización del trauma en lugar de cumplir su función terapéutica reactualiza la violencia, la presentifica, la reinstraura en la vivencia del sujeto de testimonio. Hasta que punto, en el testimonio, al reconstruir la memoria no se sujeta a las funciones narrativas víctimafvictimario, y hace del sujeto un posicionamiento contrario a la superación del acontecimiento límite. Y esta sugerencia surge precisamente porque dentro de las dimensiones de la vida, de las pulsiones, de esa fuerza irascible e indescodificable del goce, no distinguimos los límites de lo humano y lo animal; hasta el punto que la identificación con la víctima (en el sujeto mismo) podría trasgredir las propias pulsiones vitales, condenar a su patoligización los instintos profundos de nuestra vida biológica.

\section{Agresión: pulsión básica e instinto vital}

Agresión es el concepto biológico que considera el "instinto que lleva al hombre como al animal a combatir contra los miembros de su misma especie" (Lorenz 2005, p. 3). Según el zoólogo Konrad Lorenz la 
agresión es un instinto como cualquier otro, cuya función es la conservación de la vida y la especie bajo condiciones normales; el ser humano que modifica su medio vertiginosamente ha llegado por esa vía a resultados desastrosos (p. 4). Sin embargo, para Lorenz esta vía de conocimiento abre la posibilidad de entender la cultura como un sistema más complejo por medio de la observación y la inducción del comportamiento, donde los instintos que comparte con el animal representan una dimensión más simple. La etología es la ciencia que el autor citado propone con base en sus observaciones, a las que no atribuye ninguna preconcepción, por el contrario, la inducción posterior a los hechos será aquélla que pueda formular una ley de relación que permita conocer los comportamientos animales en el humano (p. 5).

Lo interesante del concepto de agresión de Lorenz tiene que ver la función específica de pervivencia del individuo y consecuentemente de la especie; esta función, como muchas otras, es el instinto. El instinto considerado así tiene como fin la adaptación al medio (Umwelt), la vida del organismo está estructurada a partir de su pervivencia en el medio, ése es el fin, el elemento a observar es la agresión, un instinto que se manifiesta en la conducta de atacar a un miembro de la misma especie; hasta aquí no hay valoraciones morales sino definiciones de orden sistémico, de tal modo que la conducta considerada instintiva sea observada en varías especies bajo determinadas circunstancias que tienen que ser descritas de igual modo. No se presentan igualdad de casos en las múltiples especies, ni tampoco en las diferentes situaciones (lucha por el territorio, por la hembra o por la ausencia o atrofia de funciones vitales), sin embargo, la explicación de la conducta sobre la base de un instinto funcional constituye la estructuración de las conductas animales sobre un orden explicativo de forma no causal sino compleja.

La noción de instinto aunque problemática guarda una pertinencia en la zoología que valdrá la pena anotar de manera sucinta, a continuación. El instinto es entendido por los biólogos que estudian el comportamiento animal a partir de las funciones vitales que el individuo de una especie presenta para mantenerse vivo; la alimentación, la respiración, la reproducción sexual son instintos en tanto estas conductas se desarrollan y realizan por medio de las partes del organismos sin ningún tipo de intencionalidad consciente; las disposiciones celu- 
lares, fisiológicas del organismo desarrollan estas disposiciones en el organismo a partir del ambiente (Umwelt).

El ambiente o medio circundante (Umwelt), en la biología de la conducta animal, fue definido por Johannes Jakob von Uexküll como la conjunción de los instrumentos o herramientas preceptúales y efectoras de un organismo vivo; estas herramientas constituyen una interacción permanente con los objetos a partir de signos (von Uexküll 1964, p. 9). El ambiente estaría integrado tanto por las herramientas del sujeto como los signos a partir de los cuales se relaciona con sus objetos (p.12); esta objetividad pertenece al mundo subjetivo del organismo viviente ya que sólo forma parte de su horizonte en tanto los signos tengan pertinencia y correlación con los instrumentos preceptúales y efectores del sujeto (p. 6). Con esta tesis, von Uexküll propone infinidad de mundos que son los ambientes (Umwelten) a partir de los cuales se organiza la vida del sujeto, cada sujeto según sus disposiciones posee su propio mundo, las disposiciones o herramientas interactúan en una remisión de signos (preceptúales y efectores) constitutivos de cada subjetividad particular (p. 5). El uso de estos instrumentos o herramientas, sobre la base de mantener la vida (pues el sujeto está definido en tanto animal como ser viviente de forma eminente), correspondería en términos de hechos observables a la conducta instintiva animal (1964).

A lo anterior, habría que agregar que según la constitución del ambiente (Umwelt) el tipo de interacción que representa el instinto estaría definido por un orden sistemático; las respuestas del sujeto a partir de los signos, descifrables según sus órganos o herramientas, estarían armonizadas como una totalidad singular en el mismo medio circundante. Pero, de igual modo dicho sistema, en el individuo viviente, sería abierto a una exterioridad y no estaría cerrado a sus meras funciones; es decir, el organismo vivo del sujeto es un sistema cuasi estable en sus funciones pero abierto a las señales de los objetos con los que interactúan y constituyen su mundo. De este modo, el organismo mantiene sus funciones con la regularidad que define su carácter normalizador: los periodos de respiración, digestión etc. Regularidades propias del individuo que se ven afectadas por la alteración de su ambiente o medio circundante, en ambos casos: Umwelt.

De igual modo, la agresión considerada como un instinto tenderá a actuar según las señales que el medio provea al organismo; la informa- 
ción que este reciba condicionará la realización de ciertas funciones o su cancelación. Casos límite como la muerte de crías provocadas por sus progenitoras son ejemplares; según Lorenz, las crías emiten signos de identificación que despiertan en la progenitora mecanismos de inhibición de la agresividad (instintos contra instintos), pero dado que algunos individuos carecen de ciertos órganos (ceguera, sordera, deficiencia de olfato) las señales de las crías no pueden ser incorporadas en el ambiente de la progenitora, de ahí que su instinto de agresión se realiza en tanto detecta un intruso en el "nido", imposibilitada de identificarlo como su cría (Lorenz 2005, p. 128-133), procede a defender el "hogar" y atacarlo hasta provocar su muerte. Esto es, "nido" y "hogar" son interpretaciones del ambiente (Umwelt) constituido por las funciones de defensa de las crías a través de la agresión de cualquier agente invasor o, en su defecto, de cualquier agente no identificado como cría.

Hasta aquí, tomado en consideración lo expuesto, podemos determinar que las funciones vitales, también consideradas como instintos y que constituyen la regularidad vital del individuo viviente son consideradas partes constitutivas de su ser. Esto significa que la respiración, la alimentación, la procreación y la agresión, entre otras, como funciones vitales son los elementos constitutivos de la vida de cada individuo; son imprescindibles para la subsistencia de la especie pero sobre todo son su naturaleza misma. Inhibir por medios artificiales dichas conductas, por ejemplo la agresión, es ir contra natura, es violentar la vida misma.

\section{Violencia: Humana demasiado humana}

Sobre la violencia habría que comenzar por distinguirla de la agresión. Para el filósofo Walter Benjamin, ésta sólo es considerada como tal cuando incide sobre relaciones morales, y la esfera propia de estas relaciones, su medio circundante (Umwelt) estarían definidos por el derecho y la justicia (Benjamin, p. 2). De aquí que podamos afirmar que a diferencia de la agresión, la violencia es humana, su medio pertenece al mundo de la vida (Lebenswelt) y por lo tanto al sistema de valores que conforman la cultura. Para Cassirer, por ejemplo, refiriéndose a la diferencia con el medio de von Uexküll, las respuestas humanas a diferencia de las animales no son inmediatas, pasan por un proceso de pensamiento que las retarda, no es una mera reacción al medio físico 
donde se desarrolla anatómicamente la experiencia del ser humano sino que éste está inmerso en una urdimbre que se consolida con la experiencia humana; el ser humano está inserto en un universo simbólico que constituye su particular modo de experimentar(se) (Cassirer 2010, p. 27).

Regresando a Benjamin, la violencia se constituye dentro de una esfera de relaciones sociales, los actos considerados violentos, la causa eficiente como él la llama, se adjudican de tal modo dentro de un marco normativo (Benjamin Op. Cit. p. 2 y ss). Y corresponde a la esfera del derecho donde se codifica la violencia en primera instancia dentro del ordenamiento entre medios y fines (p. 3). La violencia dentro del marco jurídico sólo puede ser un medio y no un fin en sí mismo; parecería entonces que la violencia es un derecho o se define como tal en cuanto al fin para el que sería utilizada, si este es justo o injusto (p. 3). Pero este principio dogmático resulta insuficiente:

En un sistema de fines justos, las bases para su crítica [de la violencia] estarían ya dadas implícitamente. Pero las cosas no son así. Pues lo que este sistema nos daría, si se hallara más allá de toda duda, no es un criterio de la violencia misma como principio, sino un criterio respecto a los casos de su aplicación (p. 2).

Para Benjamin, la violencia como principio es moral, la indagación por un criterio que defina la esencia de la violencia debe considerar a la esfera del derecho pero el criterio instrumental que la define como medio resulta insuficiente. "Pero para decidir respecto a este problema se necesita un criterio más pertinente, una distinción en la esfera misma de los medios, sin tener en cuenta los fines a los que éstos sirven" (p. 3).

La violencia como principio moral refiere a que la definición recae en el mundo de lo humano, en la interpretación de las acciones; el marco jurídico es condición necesaria pero no suficiente para su captación. En cuanto a la definición de la violencia como medio, cuyo criterio de aplicación podría definirse a partir de los fines, como empleo justo o injusto de la misma, Benjamin ve un dogma fundamental compartido tanto por el iusnaturalismo como la teoría positiva del derecho, pero encuentra problemático que la esencia de esta acción moral recaiga sólo a partir de sus fines, esto es, que su definición esté dada 
de forma extrínseca. La indagación sobre la esencia de la violencia si bien pasa por la esfera jurídica no se queda ahí, se realiza una elección, pues si la definición iusnaturalista establece que la violencia es un acto propio al individuo, tanto como dirigirse a un sitio, el derecho positivo establece una tipología de los diversos actos considerados violencia en los que cabe su legitimidad o ilegitimidad; mientras el primero sólo sanciona por los fines, el segundo establece distinciones de los actos a partir de los fines (p. 4 y ss).

La indagación por vía del derecho positivo, de igual manera, abre la posibilidad de la indagación histórica; la violencia sancionada, codificada como medio para mantener los fines jurídicos se distingue de la violencia no sancionada, de ahí que en la revisión jurídica permanezca un remanente inabarcable por semejante (Íbid). Sin embargo, en la revisión de la violencia sancionada como legítima se establece una distinción importante: la que es empleada para mantener el orden jurídico y aquella que es generadora de derecho. En ambos casos, la violencia es instrumento de coacción; sin embargo, la distinción entre el significado del uso legítimo e ilegítimo de la violencia no es evidente sin más (Íbid).

Toda violencia es, como medio, poder que funda o conserva el derecho. $\mathrm{Si}$ no aspira a ninguno de estos dos atributos, renuncia por sí misma a toda validez. Pero de ello se desprende que toda violencia como medio, incluso en el caso más favorable se halla sometida a la problematicidad del derecho en general (p. 9).

La validez de la violencia como medio está inscrita en la esfera jurídica, el problema de su aplicación se encuentra situado en el marco de la acción del poder conferido por las leyes, del contrato. La violencia es el fundamento del poder político, del Estado, el contrato originario que se hace valer por la potestad de ser la fuente de legitimidad y validez de su aplicación; de ahí que "el derecho considera la violencia en manos de la persona aislada como un riesgo o una amenaza de perturbación para el ordenamiento jurídico” (p. 6). De tal modo que el interés del derecho por monopolizar la violencia no tenga la intención de anteponer fines jurídicos o legítimos sino salvaguardarse a sí mismo; la violencia como fuente de derecho es también el instrumento del derecho para su preservación (p. 10 y ss) el Estado de derecho es 
la violencia actual o potencial ejercida sobre el individuo, expropiada al sujeto o súbdito de derecho cuya naturaleza biológica incluye la agresión como función de defensa.

La función de la violencia en la creación jurídica es, en efecto, doble en el sentido de que la creación jurídica, si bien persigue lo que es instaurado como derecho, como fin, con la violencia como medio, sin embargo - en el acto de fundar como derecho el fin perseguido- no depone en modo alguno la violencia, sino que sólo ahora hace de ella en sentido estricto, es decir inmediatamente, violencia creadora de derecho, en cuanto instaura como derecho, con el nombre de poder, no ya un fin inmune e independiente de la violencia, sino íntima y necesariamente ligado a ésta.

Los fines jurídicos son coactivos, su medio es la violencia y su origen la violencia. El derecho es violencia y busca monopolizar todo conato que ponga en riesgo su potestad, toda violencia no legitimada por sus fines es violencia contra el Estado, contra el derecho, es una amenaza y justifica el uso de la violencia validada por las leyes. Benjamin llama a este tipo de violencia que funda derecho, violencia mítica.

[T] oda violencia conservadora debilita a la larga indirectamente, mediante la represión de las fuerzas hostiles, la violencia creadora que se halla representada en ella[... ] Ello dura hasta el momento en el cual nuevas fuerzas, o aquellas antes oprimidas, predominan sobre la violencia que hasta entonces había fundado el derecho y fundan así un nuevo derecho destinado a una nueva decadencia. Sobre la interrupción de este ciclo que se desarrolla en el ámbito de las formas míticas del derecho sobre la destitución del derecho junto con las fuerzas en las cuales se apoya, al igual que ellas en él, es decir, en definitiva del estado, se basa una nueva época histórica (pp. 17-18).

La violencia mítica está condenada a cumplir la fatalidad de sus leyes históricas; cíclicas como el mito, la violencia fundante permanecerá como el eterno retorno de lo mismo pero las articulaciones jurídicas surgirán, se impondrán y se derrumbarán. Para Benjamín, la superación de la condición mítica de la violencia se da en un nivel distinto, al que llama violencia divina (p. 16 y ss); a continuación, me limitaré a sacar las conclusiones de lo enunciado anteriormente en un plano más terrenal. 


\section{Reflexiones finales}

En el presente trabajo hemos distinguido entre agresión y violencia. La agresión es una función natural de todo organismo vivo, pertenece al instinto de supervivencia y no se trata de una conducta subyacente con reglas fijas de su acción, sino de respuestas frente a estímulos cambiantes que son capaces de ser interpretados por el diseño orgánico del viviente. La agresión resulta un elemento vital insustituible como la bebida, la comida y el sexo y resulta tan antinatural privarse de aquélla como de estos. La violencia, en cambio, incide en el mundo humano, en la esfera de las relaciones sociales del derecho y la justicia. La esfera del derecho tiende a institucionalizar la violencia de forma auto conservadora para terminar decayendo al sofocar la fuente de su origen; en su intento por monopolizar la violencia, por reforzar su poder, la instituciones jurídicas se auto desenmascaran en su cruda esencia como violencia. Violencia que combate violencia despoja al derecho de su manto sagrado, pacifista y contractualista; derecho que proscribe la violencia como solución de conflictos es aquél que debilita la propia fuente de su poder: la ley cíclica del mito del poder se cumple.

En el estado actual que vivimos, no es cosa baladí traer las reflexiones de un joven Walter Benjamin; y notar que precisamente asistimos a una época en que el Estado de Derecho se desmorona en sus presupuestos míticos, portadores de legitimidad, cuando se desnuda su incapacidad de resguardar la seguridad de sus ciudadanos por medio del uso de la violencia; se desnuda porque además de ser incompetente en el uso de la violencia nuevos grupos le detenta el poder con violencia. Pero por otro lado, en el plano moral, aquél otro componente que es la sociedad civil, se muestra temerosa de aceptar la condición violenta como esencia fundamental de su contrato colectivo como Estado, e intenta combatir la violencia menguando sus propias fuerzas, descalificando a la violencia, suplicando al Estado surgiendo de la vio-

lencia que proteja sus vidas con violencia. Que aísle la violencia con violencia como medio para la paz y seguridad.

\section{Referencias}

Benjamin, W, Para una crítica de la violencia, edición electrónica: www.philosophia.cl /Escuela de Filosofía Universidad ARCIS. 
Cassirer, E., 2010, Antrpología filosófica, Fondo de Cultura Económica, México. LaCapra, D., 2009, Historia y memoria después de Auschwitz, Prometeo Libros, Buenos Aires.

—_, 2016, La historia y sus limites. Humano, animal, violencia, Bellaterra, Barcelona.

Lorenz, K., 2005, Sobre la agresión: el pretendido mal, Siglo xxi editores, México. Schiller, C. H. (ed.), 1964, Instinticve behavior. The development of a modern concept. International Universities Press, Connecticut.

Von Uexküll, J. J., 1964, "A stroll through the world of animals and men. A picture book of invisible world" en Schiller (ed.) 1964. 